\title{
ANALYTIC MAPPINGS ON HYPERFINITE SETS
}

\author{
C. WARD HENSON AND DAVID ROSS
}

(Communicated by Andreas R. Blass)

\begin{abstract}
Let $S$ and $T$ be hyperfinite sets in an $\aleph_{1}$-saturated nonstandard universe. The following are equivalent:

(i) $\frac{|S|}{|T|} \approx 1$.

(ii) There is a bijection from $S$ onto $T$ whose graph is Borel (over the internal subsets of $S \times T)$.

This follows from somewhat more general results about analytic partial functions on hyperfinite sets, the proofs of which use Choquet's theorem on the capacitibility of analytic sets.

This paper includes: proofs of the above results; an elementary direct construction for extensions of internal set functions to capacities; and a surprising corollary asserting the nonexistence of ergodic Borel transformations of a Loeb probability space.
\end{abstract}

\section{INTRODUCTION}

This paper is a contribution to a relatively new subject, descriptive set theory on hyperfinite sets, whose importance derives especially from its relation to the Loeb measure construction in nonstandard analysis. The necessary background concerning this subject can be found in [9]. The specific problem that motivated the research that is presented here is the following: Let $S$ and $T$ be hyperfinite sets in an $\aleph_{1}$-saturated nonstandard universe. (We always assume that hyperfinite sets are externally infinite in this paper.) When does there exist a bijection from $S$ onto $T$ whose graph is a nicely definable subset of $S \times T$ ? For example, when can the graph of such a bijection be taken to be a Borel set? Some limiting results are proved in [9]. For example, if there is a bijection from $S$ onto $T$ whose graph is a projective subset of $S \times T$ (or, more generally, a countably determined set), then the internal cardinalities of $S$ and $T$ must be of comparable size; i.e., the ratio $\operatorname{card}(T) / \operatorname{card}(S)$ must be finite and not infinitesimal. (It is also shown in [9] that if this condition is met, then there is a countably determined bijection from $S$ onto $T$; it is not known if there exists a projective bijection in this case.)

Received by the editors May 13, 1991 and, in revised form, September 26, 1991.

1991 Mathematics Subject Classification. Primary 03E15, 03H05; Secondary 04A15, 26E35, 28A05, 28A12, 28E05.

The research of the first author was partially supported by grants from the National Science Foundation. The research of the second author was partially supported by an SERC grant. 
In this paper we give a complete answer to this question in the situation where Borel bijections are considered: there exists a bijection from $S$ onto $T$ whose graph is a Borel subset of $S \times T$ if and only if the ratio $\operatorname{card}(T) / \operatorname{card}(S)$ is infinitely close to 1 (Theorem 1.3). We derive this result from a more fundamental result about analytic partial functions on hyperfinite sets (Theorem 1.2). This result asserts that such mappings can be approximated by internal mappings. It is derived from a more general result about analytic subsets of $S \times T$ (Theorem 1.1) whose proof uses a Choquet capacity argument that is interesting in its own right. In an appendix ( $\$ 3)$ we discuss the capacity construction that underlies this argument; it is a generalization of the Loeb measure construction [11]. This capacity construction has been used by the second author in earlier papers [13, 14] and its existence has also been noticed by Bertossi [2]. In the appendix we give a brief outline of a direct approach to this construction, and we point out simplifications in the construction and in its properties that are made possible by the conventional assumption that the nonstandard universe is $\aleph_{1}$-saturated.

We also derive several other applications of Theorem 1.2, including a proof that if $f$ is a permutation of a hyperfinite set and if the graph of $f$ is Borel, then $f$ cannot be an ergodic transformation with respect to any atomless Loeb probability measure on the set.

Most of the results in this paper were proved during the March 1987, Oberwolfach meeting on nonstandard analysis, and the authors are grateful for the opportunity that this meeting provided for them to work together. Subsequently the methods have been refined and generalized by Zivaljevic in his thesis [16] and in a series of papers [17-21].

Remark. If the nonstandard universe has the $\aleph_{0}$-isomorphism property [5], then for any hyperfinite sets $S, T$ there always exists a bijection $f$ from $S$ onto $T$ that preserves internal sets in the sense that

\section{$X$ is internal if and only if $f(X)$ is internal}

for every subset $X$ of $S$. In general this bijection cannot be Borel or even projective or countably determined. However, it does give an isomorphism of the entire hierarchy of Borel, projective, and countably determined subsets of $S$ onto the corresponding hierarchy over $T$. This shows that in a certain sense (which is weaker than the one considered in this paper) all hyperfinite sets give rise to the same descriptive set theoretic structure.

Our notation and basic formulation of nonstandard analysis is the usual; see $[1,8,10]$, for example. If $S$ is a hyperfinite set then $\operatorname{card}(S)$ will denote its internal cardinality. We regard internal finitely additive probability measures on $S$ as being defined on all internal subsets of $S$. Thus each such measure is given by an internal mapping from $S$ to the nonstandard real numbers $\geq 0$. If $\lambda$ is such a measure then we denote the corresponding Loeb measure [11] by $L(\lambda)$; this is a probability measure in the usual sense, defined on a $\sigma$-algebra of subsets of $S$ that includes all internal subsets of $S$. In particular, we shall make use of the Loeb counting measure on $S$; this is the measure $L(\lambda)$ where $\lambda$ is defined for every internal subset $X$ of $S$ by $\lambda(X)=\operatorname{card}(X) / \operatorname{card}(S)$.

The Borel hierarchy over the hyperfinite set $S$ is defined as follows: $\Sigma_{0}^{0}(S)=$ $\Pi_{0}^{0}(S)={ }^{*} P(S)$, the set of all internal subsets of $S$. For each countable ordinal 
number $\alpha, \Sigma_{\alpha}^{0}(S)$ is the set of all countable unions of sets, each of which is in $\Pi_{\beta}^{0}(S)$ for some $\beta<\alpha$, and $\Pi_{\alpha}^{0}(S)$ is the set of complements of sets in $\Sigma_{\alpha}^{0}(S)$. For each countable ordinal $\alpha, \Delta_{\alpha}^{0}=\Sigma_{\alpha}^{0} \cap \Pi_{\alpha}^{0}$. A subset of $S$ is a Borel set if it is in $\Sigma_{\alpha}^{0}(S)$ for some $\alpha$. Equivalently, the Borel sets are those in the smallest $\sigma$-algebra of subsets of $S$ that include the internal subsets of $S$.

The projective hierarchy over $S$ is defined as follows: The set $\Sigma_{1}^{1}(S)$ of analytic sets over $S$ is defined as the set of projections of sets that are Borel sets over $S \times T$ for some hyperfinite set $T$. (Equivalently, a subset of $S$ is analytic over $S$ if it can be obtained from a countable family of internal subsets of $S$ using the Souslin operation $[6,7,9]$.) $\Pi_{1}^{1}(S)$ is the set of complements of sets in $\Sigma_{1}^{1}(S)$. The higher levels $\Sigma_{n}^{1}(S)$ and $\Pi_{n}^{1}(S)$ of the projective hierarchy over $S$ are defined as usual (iterating the operations of projection and complementation further). See [9] for details.

A subset $X$ of $S$ is countably determined [7] if there exist countably many internal sets $\left\{A_{n} \mid n \in \mathbb{N}\right\}$ such that $X$ is in the complete Boolean algebra of subsets of $S$ that is generated by $\left\{A_{n} \mid n \in \mathbb{N}\right\}$. (In forming the complete Boolean algebra, unions and intersections of arbitrary size are allowed.) It is clear that every projective subset of $S$ is countably determined, and it is easy to show that the converse statement is false.

\section{Main Results}

Theorem 1.1. Let $S$ and $T$ be hyperfinite sets and let $F$ be an analytic subset of $S \times T$. Let $\mu$ be an internal finitely additive probability measure on $S$ and let $L(\mu)$ be the corresponding Loeb probability measure on $S$. Let $\pi: S \times T \rightarrow S$ be the coordinate projection map. Then, for each standard real number $\delta>0$, there is an internal set $E \subset F$ such that

$$
L(\mu)(\pi(E)) \geq L(\mu)(\pi(F))-\delta .
$$

Proof. Note that $\pi(F)$ must be an analytic subset of $S$ since $F$ is analytic. Therefore $\pi(F)$ is $L(\mu)$-measurable. The proof of Theorem 1.1 uses a capacity argument. For each internal subset $E$ of $S \times T$ we define $\Phi(E)=\mu(\pi(E))$. It is clear that $\Phi$ satisfies the hypotheses of Theorem 3.1, with $\Omega=S \times T$ and $\mathscr{F}={ }^{*} P(\Omega)$; an easy argument is needed to show that $\Phi$ is increasing and strongly subadditive. Therefore, we may define a Choquet capacity $I$ on $S \times T$ by setting

$$
I(X)=\inf \{\operatorname{st}(\Phi(E)) \mid X \subset E \subset S \times T \text { and } E \text { is internal }\}
$$

for every subset $X$ of $S \times T$.

If $E$ is internal and $X \subset E \subset S \times T$, then $\pi(X) \subset \pi(E)$ and, therefore,

$$
L(\mu)(\pi(X)) \leq \mathbf{s t}(\mu(\pi(E)))=\mathbf{s t}(\Phi(E)) .
$$

It follows from the definition of $I$ that $L(\mu)(\pi(X)) \leq I(X)$. By assumption $F$ is an analytic subset of $S \times T$; in the present context this is the same as saying that $F$ is $\mathscr{F}$-analytic (i.e., that $F$ can be obtained from sets in $\mathscr{F}$ by use of the Souslin operation) and, therefore, $F$ is capacitable for the capacity I. By Theorem 3.1 we have

$$
I(F)=\sup \{\operatorname{st}(\Phi(E)) \mid E \subset F \text { and } E \text { is internal }\} .
$$


Therefore, for each standard real number $\delta>0$, there exists an internal set $E \subset F$ such that $\operatorname{st}(\Phi(E)) \geq I(F)-\delta \geq L(\mu)(\pi(F))-\delta$. Since $E$ is internal, $\operatorname{st}(\Phi(E))=L(\mu)(\pi(E))$ and we have the desired inequality.

Remark. In our main application of Theorem 1.1 we have hyperfinite sets $S$, $T$ as above and we have two internal finitely additive probability measures, namely, $\mu$ (on $S$ ) and $\lambda$ (on $T$ ). Let $\pi_{1}: S \times T \rightarrow S$ and $\pi_{2}: S \times T \rightarrow T$ be the coordinate maps. Let $F$ be an analytic subset of $S \times T$. By applying Theorem 1.1 twice, one time for each measure, we can obtain for each standard real number $\delta>0$ an internal set $E \subset F$ such that

$$
L(\mu)\left(\pi_{1}(E)\right) \geq L(\mu)\left(\pi_{1}(F)\right)-\delta,
$$

and

$$
L(\lambda)\left(\pi_{2}(E)\right) \geq L(\lambda)\left(\pi_{2}(F)\right)-\delta .
$$

(If $E^{\prime} \subset F$ is obtained by applying Theorem 1.1 as stated and if $E^{\prime \prime} \subset F$ is obtained using the same argument for the measure $\lambda$ on $T$, then $E=E^{\prime} \cup E^{\prime \prime}$ will satisfy both inequalities simultaneously.)

Theorem 1.2. Let $S, T, \mu$, and $\lambda$ be as in the preceding remark. Let $A \subset S$ and let $f: A \rightarrow T$ be any function whose graph is an analytic subset of $S \times T$.

(a) There is an internal set $D \subset S$, differing from $A$ by an $L(\mu)$-null set, and an internal function $g: D \rightarrow T$ such that $f(A)$ and $g(D)$ differ by an $L(\lambda)$-null set and $f=g \quad L(\mu)$-almost everywhere on $A \cap D$.

(b) If $f$ is a 1-1 function, then $D$ and $g$ in $(A)$ can be chosen so that, in addition, $g$ is a 1-1 function and $f^{-1}=g^{-1} L(\lambda)$-almost everywhere on $f(A) \cap g(D)$.

Proof. (a) Let $F$ be the graph of $f$ and let $a=L(\mu)(A)$ and $b=L(\lambda)(f(A))$. By the remark after Theorem 1.1 there exists an increasing sequence of internal sets $G_{1} \subset G_{2} \subset \cdots \subset F$ such that for each standard natural number $n$, $L(\mu)\left(\pi_{1}\left(G_{n}\right)\right) \geq a-1 / n$ and $L(\lambda)\left(\pi_{2}\left(G_{n}\right)\right) \geq b-1 / n$. Each $G_{n}$ is the graph of an internal function $g_{n}$ that is the restriction of $f$ to some internal set $D_{n}$. The domain of $g_{n}$ has $L(\mu)$-measure $\geq a-1 / n$ and the range of $g_{n}$ has $L(\mu)$ measure $\geq b-1 / n$. Since our nonstandard model is assumed to be $\aleph_{1}$ saturated, there must exist an internal function $g: D \rightarrow T$ (for some internal set $D \subset S$ with $\mu(D) \approx a$ ) with the property that $g_{n}$ is a restriction of $g$ for every standard $n$ and such that $\lambda(g(D)) \approx b$. It follows immediately that $f$ and $g$ agree $L(\mu)$-almost everywhere on $A \cap D$ and that their ranges differ by a set of $L(\lambda)$ measure 0 , since they agree with $g_{n}$ on the domain of $g_{n}$ for each $n$.

(b) If $f$ is 1-1 then the functions $g_{n}$ will also be $1-1$, and the saturation argument in (a) can be modified to ensure that the additional requirements are also met by $g$.

The motivation for this paper came from the problem of determining when there could exist a bijection between hyperfinite sets $S$ and $T$ whose graph is Borel. The complete answer, which is contained in the following theorem, is that there exists a Borel bijection between $S$ and $T$ if and only if $\operatorname{st}[\operatorname{card}(T) / \operatorname{card}(S)]=1$. Moreover, if this condition is met, then there exists a bijection between $S$ and $T$ whose graph is a $\Delta_{2}^{0}$ subset of $S \times T$. 
Theorem 1.3. (A) Let $S$ and $T$ be hyperfinite sets and let $A$ be a subset of $S$. Let $f: A \rightarrow T$ be a function whose graph is an analytic subset of $S \times T$.

(i) if $f$ is surjective then $\operatorname{st}[\operatorname{card}(T) / \operatorname{card}(S)] \leq 1$;

(ii) if $A=S$ and $f$ is $1-1$, then $\operatorname{st}[\operatorname{card}(T) / \operatorname{card}(S)] \geq 1$.

(B) Let $S$ and $T$ be hyperfinite sets such that $\operatorname{st}[\operatorname{card}(T) / \operatorname{card}(S)]=1$. Then there is a bijection $f$ from $S$ onto $T$ whose graph is a $\Delta_{2}^{0}$ subset of $S \times T$.

Proof. (A) Let $\lambda_{S}$ and $\lambda_{T}$ be the internal counting measures on $S$ and $T$, respectively. Apply Theorem 1.2 to this situation, obtaining an internal function $g$ that maps a subset of $S$ into $T$, which agrees $L\left(\lambda_{S}\right)$-almost everywhere with $f$, and whose range differs from the range of $f$ by a set of $L\left(\lambda_{T}\right)$ measure 0 ; if $f$ is $1-1$ then $g$ should also be 1-1. An internal function $g$ defined on an internal set $D$ obviously satisfies $\operatorname{card}(D) \geq \operatorname{card}(g(D))$; if $g$ is also $1-1$ then $\operatorname{card}(D)=\operatorname{card}(g(D))$. The conclusions in part (A) follow immediately by a simple calculation.

(B) Without loss of generality we may assume that there exist $H, K \in{ }^{*} \mathbb{N}$ such that $H$ is infinite, $S=\{1,2, \ldots, H\}$, and $T=\{1,2, \ldots, H+K\}$. The hypothesis implies that $K / H$ is infinitesimal. Define $f: S \rightarrow T$ by

$$
f(x)= \begin{cases}x & \text { if } x / K \text { is finite } \\ x+K & \text { otherwise }\end{cases}
$$

It is easy to see that $f$ is a bijection and that the graph of $f$ is the union of a $\Sigma_{1}^{0}$ set and a $\Pi_{1}^{0}$ set.

Problem 1.4. For which hyperfinite sets $S$ and $T$ does there exist a bijection between $S$ and $T$ whose graph is a projective subset of $S \times T$ ? In particular, when is there a $\Pi_{1}^{1}$ bijection from $S$ onto $T$ ?

In [9] a partial answer to this problem is given: if $f: S \rightarrow T$ is a bijection such that the graph of $f$ is countably determined, then $\operatorname{card}(S) / \operatorname{card}(T)$ is finite and not infinitesimal; that is, the standard part of this ratio is defined and positive. Moreover, it is also shown in [9] that whenever the ratio $\operatorname{card}(S) / \operatorname{card}(T)$ is finite and not infinitesimal, then there must exist a bijection from $S$ onto $T$ whose graph is countably determined. Since the class of countably determined sets is much larger than the class of projective sets, this does not fully answer Problem 1.4.

A. Sochor has explained to us that a result similar to the one in the previous paragraph has been obtained by Cuda and Vopenka [3] in the context of Vopenka's Alternative Set Theory (AST) [15]. Since AST is a different formal system from the usual set theoretic framework for nonstandard analysis, one cannot convert proofs from one system directly into the other. However, the countably determined sets discussed here are quite analogous to the real classes in AST. Moreover, several results proved about real classes in [3] (see especially pp. 645-653) are similar to results about countably determined sets that are proved in [9].

Problem 1.5. Let $T$ be a hyperfinite set. For which hyperfinite sets $S$ does there exist an analytic subset $E$ of $S \times T$ such that $E$ is universal for analytic subsets of $T$ ? (Universality here means that every analytic subset of $T$ is of the form $\{t \mid(s, t) \in E\}$ for some $s \in S$.) 
Some information about Problem 1.5 is known. It follows from [9, Proposition 2.12] that if $S$ satisfies the condition in Problem 1.5, then the ratio

$$
\operatorname{card}(S) / 2^{\operatorname{card}(T)}
$$

is not infinitesimal. In the other direction, it is not hard to show that if $\operatorname{card}(S)>2^{n^{*} \operatorname{card}(T)}$ for every standard $n \in \mathbb{N}$, then there does exist an analytic subset $E$ of $S \times T$ that is universal for all analytic subsets of $T$. (Suppose $S$ satisfies these bounds. For a small enough infinite natural number $K$, let $S_{0}$ be the set of all internal functions $\tau$ from $\bigcup_{M \leq K}\{1,2, \ldots, K\}^{M}$ into ${ }^{*} P(T)$. We regard $S_{0}$ as the set of all internal trees of subsets of $T$ having depth $K$ and branching width $K$. If $K$ is small enough then $\operatorname{card}\left(S_{0}\right) \leq \operatorname{card}(S)$. Let $E \subset S_{0} \times T$ be the set of all pairs $(\tau, t)$ such that for some $\alpha: \mathbb{N} \rightarrow \mathbb{N}$, $t \in \bigcap_{n \in \mathbb{N}} \tau(\alpha(1), \ldots, \alpha(n))$. Then $E$ is analytic and the set $\{t \mid(\tau, t) \in E\}$ is exactly the analytic subset of $T$ that is obtained using the Souslin operation on the tree of internal sets obtained by restricting $\tau$ to sequences of standard natural numbers at finite depth in the tree. Hence $E$ has the desired universality.)

\section{ERGODIC TRANSFORMATIONS}

Let $\Omega$ be a hyperfinite set and let $\mu$ be an internal finitely additive probability measure defined on all internal subsets of $\Omega$. In this section we assume also that $\mu$ is atomless; that is, for every $\omega \in \Omega$ we have $\mu(\{\omega\}) \approx 0$. We consider the Loeb measure $L(\mu)$ that is obtained from $\mu . L(\Omega)$ will denote the $\sigma$-algebra of all $L(\mu)$-measurable subsets of $\Omega ; L(\Omega)$ contains ${ }^{*} P(\Omega)$ and also all $L(\mu)$ null sets.

Let $f: \Omega \rightarrow \Omega$ be a bijection that is measurable in the sense that for every internal $B \subset \Omega$, both $f(B)$ and $f^{-1}(B)$ are in $L(\Omega)$. Recall that such a function is ergodic provided that for every $B \in L(\Omega)$ with $L(\mu)(B \Delta f(B))=0$, either $L(\mu)(B)=0$ or $L(\mu)(B)=1$. The main purpose of this section is to prove that if the graph of the bijection $f$ is a Borel subset of $\Omega \times \Omega$ then $f$ is not ergodic. This is a consequence of Theorem 1.2, which asserts that such an $f$ can be nicely approximated by an internal 1-1 function, together with the fact that internal bijections cannot be ergodic.

Lemma 2.1. If $f: \Omega \rightarrow \Omega$ is an internal bijection then $f$ is not ergodic.

Proof. Since $f$ is internal and bijective, $\Omega$ can be partitioned into a hyperfinite collection of disjoint ${ }^{*}$ cycles, $\Omega=C_{0} \cup C_{1} \cup \cdots \cup C_{N}$, where each $C_{i}$ is of the form $\left\{\omega_{i}, f\left(\omega_{i}\right), f^{2}\left(\omega_{i}\right), \ldots, f^{n_{i}}\left(\omega_{i}\right)\right\}$ for some $\omega_{i} \in \Omega$ and some $n_{i} \in{ }^{*} \mathbb{N}$, and $f^{n_{i}+1}\left(\omega_{i}\right)=\omega_{i}$ for each $i=0,1, \ldots, N$.

Let us first assume that $L(\mu)\left(C_{i}\right)=0$ for all $i=0,1, \ldots, N$. Since $\mu$ is internal, there exists an $m \leq N$ such that if we set $E=C_{0} \cup C_{1} \cup \cdots \cup C_{m-1}$ then $\mu(E) \leq \frac{1}{2}<\mu\left(E \cup C_{m}\right)$. But then $f$ cannot be ergodic since $f(E)=E$ and $L(\mu)(E)=\frac{1}{2}$.

If the above case does not apply, then without loss of generality we may assume that $L(\mu)\left(C_{0}\right)>0$. Since $\mu$ is internal there must exist an $n \leq n_{0}$ such that if we set $E=\left\{\omega_{0}, f\left(\omega_{0}\right), \ldots, f^{n-1}\left(\omega_{0}\right)\right\}$ then $\mu(E) \leq \frac{1}{2} \cdot \mu\left(C_{0}\right)<$ $\mu\left(E \cup\left\{f^{n}\left(\omega_{0}\right)\right\}\right)$. In this case $L(\mu)(E)=\frac{1}{2} \cdot \operatorname{st}\left(\mu\left(C_{0}\right)\right)$, which is strictly between 0 and 1 . However, $L(\mu)(E \Delta f(E))=L(\mu)\left(\left\{\omega_{0}, f^{n}\left(\omega_{0}\right)\right\}\right)=0$, showing that $f$ is not ergodic in this case either. 
Theorem 2.2. If $f: \Omega \rightarrow \Omega$ is a bijection and if the graph of $f$ is a Borel subset of $\Omega \times \Omega$, then $f$ is not ergodic.

Proof. By Theorem 1.2, $f$ is equal $L(\mu)$-almost everywhere to an internal 1-1 function $g: D \rightarrow T$ for some internal set $D \subset \Omega$ such that $\mu(D) \approx 1$. Evidently $D$ and $g(D)$ have the same internal cardinality, so we may extend $g$ to an internal bijection from $\Omega$ onto $\Omega$. By Lemma 2.1 there exists a measurable set $E$ with $0<L(\mu)(E)<1$ and $L(\mu)(E \Delta g(E))=0$. Moreover,

$$
L(\mu)(E \Delta f(E)) \leq L(\mu)(E \delta g(E))+L(\mu)(g(E) \Delta f(E))=0,
$$

completing the proof that $f$ is not ergodic.

Problem 2.3. Does the probability space $(\Omega, L(\Omega), L(\mu))$ have any ergodic transformations?

\section{AN APPENDIX ON CAPACITIES}

Let $\Omega$ be an internal set and let $\mathscr{F}$ be an internal paving on $\Omega$; that is, $\mathscr{F}$ is an internal subset of ${ }^{*} P(\Omega)$ that contains $\varnothing$ and is closed under (standardly) finite unions and intersections. Suppose further that $\Phi: \mathscr{F} \rightarrow{ }^{*} \mathbb{R}$ is an internal mapping that is increasing and strongly subadditive. That is, for any $A, B \in \mathscr{F}$,

(i) $A \subset B$ implies $\Phi(A) \leq \Phi(B)$;

(ii) $\Phi(A \cup B)+\Phi(A \cap B) \leq \Phi(A)+\Phi(B)$.

In this situation, consider the external function $\operatorname{st}(\Phi(A))$ that maps $\mathscr{F}$ into the extended real numbers. (If $\alpha$ is an infinite number in ${ }^{*} \mathbb{R}$, then we take st $(\alpha)=+\infty$ if $\alpha>0$ and $\operatorname{st}(\alpha)=-\infty$ if $\alpha<0$.) It has been shown by Bertossi [2] and independently by the second author [14] that $\operatorname{st}(\Phi(A))$ can be extended to a Choquet $\mathscr{F}$-capacity on $\Omega$, and it is this result that we need, in a somewhat improved form, for the main argument in this paper. To prove this fact, they observe that $\operatorname{st}(\Phi(A))$ is increasing and strongly subadditive, and also that it is continuous on increasing sequences from $\mathscr{F}$ whose union is also in $\mathscr{F}$. This latter fact is an obvious consequence of the hypothesis that the nonstandard model is $\aleph_{1}$-saturated. They then apply Choquet's fundamental extension theorem (see [4] or [12]) to complete the proof.

This result and its proof generalize Loeb's [11], in which the Caratheodory extension theorem was used to extend the standard part of an internal finitely additive measure to a $\sigma$-additive measure on a $\sigma$-algebra of sets. This measure construction, which yields the class of measures that are now known as the Loeb measures, is one of the most useful and important tools in nonstandard analysis, and we believe that this Choquet capacity version of the construction will also prove to be extremely useful.

In this appendix we sketch an easier and more elementary proof that there is an $\mathscr{F}$-capacity $I$ on $\Omega$ that extends $\operatorname{st}(\Phi(A))$. An advantage of this argument is that our definition of $I$ is much simpler than the one that comes out of the general theory. We are able to work with this simpler definition (which would not be correct for the general situation) because we make fuller use of the $\aleph_{1}$ saturation hypothesis than was done in [2,14]. (For an expository treatment of the Loeb measure construction that is similar in its approach to the more general argument given in this section, see [10].)

Recall that a set $X$ is capacitable (with respect to a paving $\mathscr{F}$ and an $\mathscr{F}$ capacity $I)$ provided $I(X)$ is the supremum of the numbers $I(E)$, where $E$ 
ranges over subsets of $X$ that are countable intersections of elements of $\mathscr{F}$. Choquet's Capacitability Theorem $[4,12]$ asserts that every $\mathscr{F}$-analytic set is capacitable.

Theorem 3.1. Let $\Omega, \mathscr{F}$, and $\Phi$ be as above. For each subset $X$ of $\Omega$ (whether external or internal) define $I(X)=\inf \{\operatorname{st}(\Phi(E)) \mid X \subset E \subset \Omega$ and $E \in \mathscr{F}\}$. Then

(a) $I$ is a strongly subadditive Choquet $\mathscr{F}$-capacity on $\Omega$;

(b) if $X \subset \Omega$ is capacitable, then $I(X)=\sup \{\operatorname{st}(\Phi(E)) \mid E \subset X$ and $E \in \mathscr{F}\}$. In particular, this equation is true if $X$ is $\mathscr{F}$-analytic (i.e., $X$ can be obtained from sets in $\mathscr{F}$ by use of the Souslin operation).

Proof. (a) Note that for any $E \in \mathscr{F}, I(E)=\operatorname{st}(\Phi(E))$ since $\operatorname{st}(\Phi(E))$ is increasing on $\mathscr{F}$. Clearly $I$ is increasing, and an easy approximation argument shows that it is strongly subadditive as well.

Next we observe that if $\left\{E_{n}\right\}$ is a decreasing sequence of sets from $\mathscr{F}$, then

$$
I\left(\bigcap E_{n}\right)=\inf _{n} I\left(E_{n}\right) \text {. }
$$

Indeed, fix a standard real number $\varepsilon>0$ and choose $E \in \mathscr{F}$ that satisfies $\bigcap E_{n} \subset E$ and $I(E) \leq I\left(\cap E_{n}\right)+\varepsilon$. The $\aleph_{1}$-saturation hypothesis implies that there must exist a standard integer $m$ with $E_{m} \subset E$. Hence $\inf _{n} I\left(E_{n}\right) \leq$ $I\left(\cap E_{n}\right)+\varepsilon$. Since $\varepsilon$ was arbitrary, this proves $\inf _{n} I\left(E_{n}\right) \leq I\left(\cap E_{n}\right)$. The desired equality follows immediately because $I$ is increasing. $\Omega$,

It remains to prove that for every increasing sequence $\left\{X_{n}\right\}$ of subsets of

$$
I\left(\bigcup X_{n}\right)=\sup _{n} I\left(X_{n}\right) .
$$

Because $I$ is increasing, we need only prove the inequality $\leq$. Let $\alpha$ be the extended real number defined by setting $\alpha=\sup _{n} I\left(X_{n}\right)$. For the moment assume $-\infty<\alpha<\infty$. Let $\varepsilon$ be an arbitrary standard positive real number, and choose a strictly increasing sequence $\left\{\varepsilon_{n}\right\}$ of standard real numbers that satisfy $0<\varepsilon_{n}<\varepsilon$ for all $n \in \mathbb{N}$. Let $\delta_{n}=\varepsilon_{n}-\varepsilon_{n-1}$ for each $n$. Choose $F_{n} \in \mathscr{F}$ so that $X_{n} \subset F_{n}$ and $I\left(F_{n}\right) \leq I\left(X_{n}\right)+\delta_{n}$ for each $n$. Put $E_{n}=F_{1} \cup \cdots \cup F_{n}$ so that $\left\{E_{n}\right\}$ is an increasing sequence and $X_{n} \subset E_{n}$ for each $n$. One proves easily by induction on $n$ that $I\left(E_{n}\right) \leq I\left(X_{n}\right)+\varepsilon_{n}$. By the $\aleph_{1}$-saturation hypothesis, we may extend $\left\{E_{n} \mid n \in \mathbb{N}\right\}$ to an internal sequence $\left\{E_{n} \mid 1 \leq n \leq H\right\}$ for $H$ an infinite integer. Without loss of generality we may assume that $E_{n} \in \mathscr{F}$ for all $n$ and that $E_{n} \subset E_{m}$ for all $n \leq m$ (with $1 \leq n \leq m \leq H$ ).

Note that for all standard integers $n$ we have

$$
\operatorname{st}\left(\Phi\left(E_{n}\right)\right)=I\left(E_{n}\right) \leq I\left(X_{n}\right)+\varepsilon_{n} \leq \alpha+\varepsilon_{n}<\alpha+\varepsilon .
$$

Since $\alpha+\varepsilon$ is a standard real number, this implies that $\Phi\left(E_{n}\right)<\alpha+\varepsilon$ for all standard $n$. Hence there exists an infinite integer $N \leq H$ so that $\Phi\left(E_{n}\right)<\alpha+\varepsilon$ holds for all $n$ in the interval $1 \leq n \leq N$. But then $E=E_{N}$ is in $\mathscr{F}$ and satisfies $\bigcup X_{n} \subset E$ and $\Phi(E)<\alpha+\varepsilon$. This shows that $I\left(\bigcup X_{n}\right)<\alpha+\varepsilon$ for every standard $\varepsilon>0$, and hence

$$
I\left(\bigcup X_{n}\right) \leq \alpha=\sup _{n} I\left(X_{n}\right) \text {. }
$$


If $\alpha=\infty$, there is nothing to prove. If $\alpha=-\infty$, then a slight modification of the saturation argument just given is needed. This completes the proof that $I$ is an $\mathscr{F}$-capacity on $\Omega$.

(b) Suppose that $X$ is a capacitable subset of $\Omega$. Capacitability of $X$ means that for each standard real number $\alpha<I(X)$ there exists a decreasing sequence of sets $\left\{E_{n} \mid n \in \mathbb{N}\right\} \subset \mathscr{F}$ that satisfies $\bigcap E_{n} \subset X$ and $\alpha<I\left(\bigcap E_{n}\right)$. The $\aleph_{1}$-saturation hypothesis implies that we may extend the given sequence to an internal sequence $\left\{E_{n} \mid 1 \leq n \leq H\right\}$ in $\mathscr{F}$, where $H$ is an infinite integer. By decreasing $H$ if necessary (but keeping $H$ infinite) we may ensure that $E_{n} \supset E_{m}$ whenever $1 \leq n \leq m \leq H$. By the choice of $\left\{E_{n}\right\}$ we know that $\alpha<I\left(E_{n}\right)=\operatorname{st}\left(\Phi\left(E_{n}\right)\right)$ and hence $\alpha<\Phi\left(E_{n}\right)$ for all standard $n \in \mathbb{N}$. Hence there exists an infinite integer $N \leq H$ such that $\alpha<\Phi\left(E_{N}\right)$. Then $E_{N} \subset \bigcap\left\{E_{n} \mid n \in \mathbb{N}\right\} \subset X$ and $\alpha \leq \operatorname{st}\left(\Phi\left(E_{N}\right)\right)=I\left(E_{N}\right)$. Since $\alpha$ was an arbitrary standard real number $<I(X)$, this shows that $X$ can be approximated from within by sets from $\mathscr{F}$ in the desired way. (In the trivial case where $I(X)=-\infty$, we just approximate $X$ from inside by $\varnothing$.) For the last statement in (b), we note that it is well known that all $\mathscr{F}$-analytic sets are capacitable for any Choquet $\mathscr{F}$-capacity (see $[4,12])$.

\section{REFERENCES}

1. S. Albeverio, J.-E. Fenstad, R. Hoegh-Krohn, and T. Lindstrom, Nonstandard methods in stochastic analysis and mathematical physics, Academic Press, New York, 1986.

2. L. E. Bertossi, Standard capacities with nonstandard pavings, Notas Soc. Mat. Chile 5 (1986), 1-6.

3. K. Cuda and P. Vopenka, Real and imaginary classes in the Alternative Set Theory, Comment. Math. Univ. Carolin. 20 (1979), 639-653.

4. C. Dellacherie and P. A. Meyer, Probabilities and potential, North-Holland, Amsterdam, 1978.

5. C. W. Henson, The isomorphism property in nonstandard analysis and its use in the theory of Banach spaces, J. Symbolic Logic 39 (1974), 717-731.

6. __ Analytic sets, Baire sets, and the standard part map, Canad. J. Math. 31 (1979), 663-672.

7. __ Unbounded Loeb measures, Proc. Amer. Math. Soc. 74 (1979), 143-150.

8. A. E. Hurd and P. A. Loeb, An introduction to nonstandard real analysis, Academic Press, New York, 1985.

9. H. J. Keisler, K. Kunen, A. Miller, and S. Leth, Descriptive set theory over hyperfinite sets, J. Symbolic Logic 54 (1989), 1167-1180.

10. T. Lindstrom, An invitation to nonstandard analysis, Nonstandard Analysis and its Application (N. Cutland, ed.), London Math. Soc. Stud. Texts, vol. 10, Cambridge Univ. Press, Cambridge and New York, 1988, pp. 1-105.

11. P. A. Loeb, Conversion from nonstandard to standard measure spaces and applications in probability theory, Trans. Amer. Math. Soc. 211 (1975), 113-122.

12. P. A. Meyer, Probability and potentials, Blaisdell, Waltham, MA, 1966.

13. D. A. Ross, Measurable transformations in saturated models of analysis, Ph.D. thesis, Univ. of Wisconsin at Madison, 1983.

14. __ Lifting theorems in nonstandard measure theory, Proc. Amer. Math. Soc. 109 (1990), 809-822.

15. P. Vopenka, Mathematics in the Alternative Set Theory, Teubner-Texte, Leipzig, 1979.

16. B. Zivaljevic, Hyperfinite transversal theory, Ph.D. thesis, Univ. of Illinois at UrbanaChampaign, 1989. 
17. __ Some results about Borel sets in descriptive set theory of hyperfinite sets, J. Symbolic Logic 55 (1990), 604-614.

18. $\ldots$, The structure of graphs all of whose Y-sections are internal sets, J. Symbolic Logic 56 (1991), 50-66.

19.,$U$-meager sets when the cofinality and the coinitiality of $U$ are both uncountable, J. Symbolic Logic 56 (1991), 906-914.

20. __ Every Borel function is monotone Borel, Ann. Pure Appl. Logic 54 (1991), 87-99.

21. __ Uniqueness of unbounded Loeb measure using Choquet's theory, Proc. Amer. Math. Soc. 116 (1992), 529-533.

Department of Mathematics, University of Illinois, 1409 West Green Street, Urbana, ILLINOIS 61901

E-mail address: henson@symcom.math.uiuc.edu

Department of Statistics, 44 Kidder Hall, Oregon State University, Corvallis, OREGON 97331

Current address, David Ross: Department of Mathematics, University of Hawaii at Manoa, 2565 The Mall, Honolulu, Hawaii 96822

E-mail address: ross@kahuna.math.hawaii.edu 